\title{
COMPARATIVE ANALYSIS OF NATIONAL OPEN DATA PORTALS OR WHETHER YOUR PORTAL IS READY TO BRING BENEFITS FROM OPEN DATA
}

\author{
Anastasija Nikiforova \\ Faculty of Computing, University of Latvia \\ Rainis Boulevard 19. Riga, LV-1586, Latvia
}

\begin{abstract}
This paper focuses on the analysis of usability of the national open data portals. Open [government] data are considered as one of the most influenceable tool for preventing and reducing corruption and reaching innovative solutions that create added value for society. Thus, it is important to ensure that they are provided in a form that are useful and suitable for the original purpose of the open data. Critical voices and many discussions on whether open government data and national open data portals are of sufficient quality appear more frequently. Therefore, this study deals with this topic and aims to find the main challenges that can negatively impact users' experience through an analysis of usability of 42 open data portals by applying a unified methodology on them allowing their comparative analysis to be carried out. This study highlights the weakest aspects for 42 national open data portals, pointing on both, the most common weakest points, and individual. The analysis carried out also identifies portals that can be considered as leaders and as an example for the less successful open data portals.
\end{abstract}

\section{KEYWORDS}

Open Data, Open Data Government, Open Data Portal, Usability of a Portal

\section{INTRODUCTION}

Open government data (OGD) initiative is an approach in which external stakeholders can play a greater role in the innovation of government services, providing citizens with new and innovative services, and have the capacity to play a catalytic role in co-creation of new public services (McBride et al., 2018). OGD also becomes the main topic of the 2030 Agenda for Sustainable Development (UNDESA, 2016). However, it is known that open data itself does not play an important role since value is generated by the use of data (Charalabidis et al., 2018). Therefore, barriers should be identified and solved.

According to (McBride et al., 2018), the most of OGD barriers are directly related to the release and publishing of open data, while at the user level, most of the barriers relate to their ability to use OGD, which is related to open data portals and, in particular, their usability. This is also in line with (Janssen et al., 2012), according to which, a sufficiently high-quality and user-friendly open data portal are needed, which allow access to the data for any stakeholder, resulting in the transformation of the data into a value for the society (Faini and Palmirani, 2016). While previous studies deal with quality of open data (Nikiforova, 2019, 2020b), this paper addresses this issue by focusing on the open data portal. One of the most important reasons for initiating such research is critical voices, which appear more frequently, addressing the current issue of the low quality of open data portals, which is a serious risk that can disrupt the open data project (Umbrich et al., 2015).

The aim of the study is to explore 42 national open data portals, by evaluating them from the perspective of the usability in order to understand the current state of national open data portals and compare them, trying to identify (a) key challenges and (b) portals which can be used as an example by other less competitive portals. As for the method used, the preference was given to the framework proposed in (Máchová et al., 2018) as one of the most recent and cited in the last 5 years. Such an assessment can be used to compare different portals, thereby helping governments and citizens to understand where the country is between other 
countries in the scope of certain aspects. Among other results, this study outlines the progress of national open data portals.

The paper addresses the following issues: a brief overview of the related researches (Section 2), an overview of the current state of national open data portals and rationale for research (Section 3), an analysis of 42 national open data portals, highlighting their main challenges (Section 4), and conclusions (Section 5).

\section{RELATED WORKS}

Although the popularity of the open data is increasing more rapidly, that is also reflected in the number of studies covering this topic, only a few studies cover the topic of open data portal. According to Google Scholar data analysed, despite the increase in popularity of the topics of the OGD and open data portals, the ratio of these researches to the researches dealing with open data does not exceed 7\% (Nikiforova, 2020a). The usability of open data portals is being studied even less frequently, since over the last 5 years, only $6 \%$ of studies covering open data portal topic at least a little, mention the usability aspect. It also demonstrates the "uniqueness" of the presented research. However, it should be also mentioned that a number of benchmarks and indexes were recently introduced to assess the state of OGD and open data portals (a brief overview in (Nikiforova, 2020a)).

According to (Attard et al., 2015) and the author of this study, many researchers cover their own national OGD initiatives, which in most cases turns to the an assessment of national open data portals, assessing them from different perspectives, including aspects such as data, functionality, features, stakeholder participation, stakeholder feedback. Other studies focus on such aspects as the relevance of the open data sets of a specific portal to the "5-stars" classification (Matheus et al., 2012).

According to (Osagie et al., 2017), most of the studies focus mainly on data delivery and the data environment, considering what data providers have done to facilitate users, but have not actually consulted users. Thus, it appears that most of the frameworks lack a user perspective. In addition, most studies (a) mainly refer to one or just only a few countries without a comprehensive comparison between different initiatives, (b) lack a common methodology that would allow comparisons between studies and portals.

The study that drew the attention of the author of this paper to base the study on, is the framework proposed in (Máchová et al., 2018). This study proposes to evaluate open data portals using three key aspects, divided into 14 sub-aspects: (1) open data specification, which includes (a) description of data set, (b) publisher of data set, (c) thematic categories and tags, (d) release date and up to date, (e) machine readable formats, $(f)$ open data licence, $(g)$ visualization and analytics tools; (2) open data set feedback, which includes (a) documentation and tutorials, (b) forum and contact form, (c) user rating and comments, (d) social media and sharing; (3) open data set request, which includes such aspects as (a) request form, (b) list of requests, (c) involvement in the process. According to the authors and studies underpinning this methodology, these 14 criteria reflect all the functionality of the portal and are typical tasks normally performed by users on the portal. In addition, it complies with the majority principles used or mentioned by other well-known researchers (see (Charalabidis et al., 2018), (Attard et al., 2015), (Zuiderwijk et al., 2015)). This methodology was found to be reasonably appropriate including the fact that it is cited by other researchers, meaning that it is accepted by the public/ researchers. It also considers a user perspective that, according to (Osagie et al., 2017), is one of the key challenges for most solutions. While (Máchová et al., 2018) analysed open data portals of Canada, Australia, India, UK, USA, this paper extends this significantly, by applying the methodology to 42 portals that allows to carry out comprehensive analysis, providing their comparison. Thus, common challenges and "good examples" are identified.

\section{OPEN DATA PORTALS AND RATIONALE FOR THE RESEARCH}

Nowadays, open data can be considered as a major step towards a digital society (Hansen and Schrøder, 2019). Many countries have established open data portals in order to provide access to open data sets, which allows public sector information to be opened to maximise their re-use by collecting and publishing different data catalogues to a wide audience for their further reuse (Attard et al., 2015). The development of software 
applications and services through open data provides many benefits to citizens and society as a whole, such as greater transparency, communication, acquisition of trust, innovation, and business value.

However, despite the fact that many countries develop and launch their own open data portals, they received a great deal of criticism from both, society by which is meant users who may not have IT knowledge, and technical experts. In other words, despite the fact that open data portals are intended to benefit and improve the re-use of open data and exploit its potential, the more often they do not achieve these aims and the poor usability of the open data portals is highlighted by complaining that these platforms were not intended for non-technical users ((Osagie et al., 2017), (Tinholt et al., 2013), (Purwanto et al., 2020)). The European Data Portal (Blank, 2019) and (Tinholt, 2013) also indicate that the usability is one of the main aspects of open data portals (among data availability, political leadership, and data portal usability). It follows from all the above that the quality of open data portals and, in particular, their usability are vital. This study deals with this issue. At present, one of the most comprehensive and up-to-date reports on open data portals is the European Data Portal report (Blank, 2019), which evaluates EU28+ national open data portals, assessing policy, portal, impact, and quality. As for the portal category, it is divided into 4 groups: feature, usage, data provision and sustainability.

Table 1 summarises the development of open data portals over the years (2016-2019) and ranking evolution ("up" and "down" arrows), based on the annual reports published by the European Data Portal. It shows whether and how fast portals are developing, what are the leading countries and which countries are less successful.

In 2019 (Blank, 2019) analysed 32 national open data portals and, according to Table 1, the best results were demonstrated by France, Spain, and Ireland. In terms of progress, the best progress in one year was demonstrated by Cyprus, which took $24^{\text {th }}$ place in 2017 , however came $2^{\text {nd }}$ in 2018 , but the most impressive negative result was demonstrated by Germany, which lost 23 positions between 2017 and 2018. Moreover, only four countries improve their positions from year to year - Ireland, Latvia, Italy, and Malta. The most impressive result in terms of continuous development was demonstrated by Latvia, which improves its position from the first appearance in this rating.

Table 1 . The maturity of open data portals

\begin{tabular}{|c|c|c|c|c|c|c|c|c|c|}
\hline Country & 2019 & 2018 & 2017 & 2016 & Country & 2019 & 2018 & 2017 & 2016 \\
\hline France & $1 \uparrow+4$ & $5 \uparrow+3$ & $8 \downarrow-5$ & 3 & Belgium & $17 \downarrow-2$ & $15 \uparrow+3$ & $12 \downarrow-1$ & 11 \\
\hline Spain & $2 \uparrow+6$ & $8 \downarrow-3$ & $5 \downarrow-1$ & 4 & Estonia & $18 \uparrow+8$ & $26 \uparrow+10$ & $16 \downarrow-14$ & 2 \\
\hline Ireland & 3 & 3 & $3 \uparrow+7$ & 10 & Denmark & $19 \uparrow+10$ & $29 \uparrow+2$ & $27 \downarrow-10$ & 17 \\
\hline Cyprus & $4 \downarrow-2$ & $2 \uparrow+22$ & $24 \downarrow-6$ & 18 & Norway & $20 \uparrow+2$ & $22 \downarrow-8$ & 14 & 14 \\
\hline Finland & $5 \downarrow-4$ & $1 \uparrow+5$ & $6 \uparrow+3$ & 9 & Bulgaria & $21 \uparrow+3$ & 18 & $18 \downarrow-10$ & 8 \\
\hline Slovenia & $6 \uparrow+1$ & $7 \uparrow+13$ & $20 \downarrow-7$ & 13 & UK & $22 \downarrow-6$ & $16 \downarrow-5$ & $11 \uparrow+5$ & 16 \\
\hline Austria & $7 \uparrow+3$ & $10 \downarrow-1$ & $9 \downarrow-4$ & 5 & Malta & $23 \uparrow+8$ & 31 & $31 \uparrow+1$ & 32 \\
\hline Romania & $8 \downarrow-4$ & 4 & $4 \uparrow+11$ & 15 & Switzerland & $24 \downarrow-7$ & $17 \uparrow+8$ & $25 \downarrow-3$ & 22 \\
\hline Luxembourg & 9 & $9 \downarrow-8$ & 1 & 1 & Portugal & $25 \downarrow-2$ & $23 \downarrow-4$ & $19 \uparrow+1$ & 20 \\
\hline Netherlands & $10 \uparrow+4$ & $14 \downarrow-4$ & $10 \downarrow-3$ & 7 & Sweden & $26 \downarrow-5$ & $21 \downarrow-4$ & $17 \uparrow+10$ & 27 \\
\hline Latvia & $11 \uparrow+8$ & $19 \uparrow+2$ & $21 \uparrow+9$ & 30 & Lithuania & 27 & $27 \uparrow+1$ & $28 \downarrow-3$ & 25 \\
\hline Poland & $12 \downarrow-1$ & $11 \uparrow+12$ & $23 \uparrow+1$ & 24 & Czech Republic & 28 & $28 \downarrow-6$ & $22 \uparrow+1$ & 23 \\
\hline Italy & 13 & 13 & $13 \uparrow+6$ & 19 & Slovakia & $29 \downarrow-15$ & $12 \uparrow+3$ & $15 \uparrow+6$ & 21 \\
\hline Germany & $14 \uparrow+9$ & $25 \downarrow-23$ & $2 \uparrow+4$ & 6 & Hungary & 30 & - & $30 \downarrow-2$ & 28 \\
\hline Greece & $15 \downarrow-9$ & $6 \uparrow+20$ & 26 & 26 & Iceland & $31 \downarrow-1$ & $30 \downarrow-1$ & 29 & 29 \\
\hline Croatia & $16 \uparrow+4$ & $20 \downarrow-13$ & $7 \uparrow+5$ & 12 & Liechtenstein & 32 & - & $32 \downarrow-1$ & 31 \\
\hline
\end{tabular}

As for the given research, a total of 40 users with IT background participated in the experiment, that corresponds to the experiment design proposed in (Máchová et al., 2018). The experiment took place from January to May 2020. This method was also chosen because the expert assessment was found to be one of the most appropriate methods for the OGD and related analyses (Susha et al., 2015). 


\section{ASSESSMENT OF OPEN DATA PORTALS}

As in (Máchová et al., 2018), a three-level Likert scale (fulfilled - 3, partially fulfilled - 2, and unfulfilled 1), that belongs to the acceptability tasks, was used to measure the usability of 42 national open data portals. Portals were selected by combining all portals addressed in (Blank, 2019), more precisely EU28+, by supplementing this list with portals originally addressed in (Máchová et al., 2018) - Canada, Australia, India, UK, USA, and some other portals on which positive feedback from their users are observed. 40 participants were selected on a voluntary basis to carry out this task, using in-group (between subject) observational method to reduce the potential subjectivity of the results, which cannot be obtained if the total number of participants is less than 30. This also means that if this experiment is replicated, the results may vary slightly, however, such a selection reduces it to a minimum and allows to carry out a comparative analysis, since all portals were assessed by the same group of participants. Each portal was analysed by assessing the fulfilment of 14 criteria representing 3 categories:

1) open data specification including such aspects as (1a) description of data set, (1) publisher of data set, (1c) thematic categories and tags, (1d) release date and up to date, (1e) machine readable formats, $(\mathbf{1 f})$ open data licence, $(\mathbf{1 g})$ visualization and analytics tools;

2) open data set feedback including (2a) documentation and tutorials, (2b) forum and contact form, (2c) user rating and comments, (2d) social media and sharing;

3) open data set request including (3a) request form, (3b) list of requests, (3c) involvement in the process.

Table 2 summarises the results of the analysis, where (a) the $1^{\text {st }}$ column is a portal ranking based on the results of the assessment ( $7^{\text {th }}$ column "Total"), (b) $3^{\text {rd }}$ column contains data regarding available languages, where the language notion corresponds with ISO 639-1 (two-letter codes), (c) $4^{\text {th }}-6^{\text {th }}$ columns represents the score for three categories ( $I$ for open data specification, $I I$ for open data set feedback and $I I I$ for open data set request) and $7^{\text {th }}$ column is a total score that can vary from 14 to 42 points for each portal. The last column ("Rank EDP") contains the rank of the portal, according to (Blank, 2019) and Table 1.

The overall result of all aspects for all countries is 29.76 out of 42 points. Since the result is far from perfect, and, this applies to all portals, as none of the portals has scored more than 38.17 points (Cyprus), it can be concluded that there is room for improvements for all portals analysed.

The first category, namely "open data set specification", appears to be the best among the assessed, since it was assessed on average by 2.38 points out of 3 (total of 16.7 out of 21), while the second - "open dataset feedback" - gained only 1.98 points (total of 7.9 out of 12), however, "open dataset request" - 1.72 points (total of 5.2 out of 9).

For the first aspect - "description of dataset", the positive point is that most open data portals meet this requirement. However, nevertheless, data publishers often do not provide any or sufficiently detailed descriptions, and in many cases only the name of data set is recopied as a description, but in some cases the specification is missing and not even intended to appear that is the case for Malta. For the best results, which ranges between 17.9 and 20.2 point out of 21, they were demonstrated by France, Cyprus, Singapore, Portugal, Austria, Slovenia, Finland, India, Spain. However, the worst results were demonstrated by Malta, Czech Republic, Poland, Croatia, Belgium, Japan, Lithuania and Romania scoring between 12.41 and 15.3 points. As regards the "publisher of dataset", this information is mandatory for most open data portals. This is also valid for the majority of the aspects of this category.

For "open dataset feedback" category, which includes documentation and tutorials, forums and contact form etc., the most successful portals are Austrian, Finnish, Russian, Taiwanese, Portuguese, Cypriot and Romanian portals, estimated between 11.59 and 10.14 out of 12 points. However, this aspect appears to be very weak for Bulgaria, Sweden, Switzerland, Denmark, Hungary, Iceland, Liechtenstein and Japan, as the overall score is below 6 points.

In scope of the category of "dataset requests", the best results were demonstrated by New Zealand, Lithuania, Russia, Colombia, Canada, Spain and Cyprus, with a score of between 8.78 and 7.34 out of 9 points. The Irish portal has to be mentioned here, as it not only allows to request a data set, but also assumes that the request can be commented, and as a result the portal's employees explain to the users whether (and why) the request can or cannot be met, which is a great opportunity because it can create feedback and educate users, especially when users do not understand what data can be opened, that is the case for Latvia (Nikiforova, 2020a). 
Table 2. Results of assessment of open data portals

\begin{tabular}{|c|c|c|c|c|c|c|c|}
\hline \# & Country & Languages & $\bar{I}$ & $\overline{I I}$ & $I I I$ & TOTAL & $\operatorname{Rank}(E D P)$ \\
\hline 1 & Cyprus & EL, EN & 20,2 & 10,63 & 7,34 & 38,17 & 4 \\
\hline 2 & Russia & RU, EN & 16,61 & 11,39 & 8,49 & 36,49 & N/A \\
\hline 3 & France & EN, FR, ES & 20,2 & 9,38 & 6,59 & 35,93 & 1 \\
\hline 4 & Spain & ES, CA, GL, EU, EN & 17,97 & 9,58 & 8,01 & 35,56 & 2 \\
\hline 5 & Taiwan & EN, TW, CN & 17,09 & 10,84 & 7,06 & 34,99 & N/A \\
\hline 6 & Canada & EN, FR & 17,57 & 8,97 & 8,22 & 34,76 & N/A \\
\hline 7 & Austria & $\mathrm{AU}$ & 18,84 & 11,59 & 4,31 & 34,74 & 7 \\
\hline 8 & Colombia & ES, EN & 17,59 & 8,36 & 8,24 & 34,19 & N/A \\
\hline 9 & New Zealand & EN & 17,67 & 7,26 & 8,78 & 33,71 & N/A \\
\hline 10 & Ireland & EN, GA & 17,7 & 8,79 & 7,09 & 33,58 & 3 \\
\hline 11 & Portugal & EN, FR, ES, PT & 19,01 & 10,69 & 3,65 & 33,35 & 25 \\
\hline 12 & Finland & FI, SV, EN & 18,7 & 10,85 & 3,35 & 32,9 & 5 \\
\hline 13 & Lithuania & $\mathrm{LT}, \mathrm{EN}$ & 15,27 & 8,19 & 8,75 & 32,21 & 27 \\
\hline 14 & Slovenia & SL +Google Translate & 18,71 & 9,15 & 4,28 & 32,14 & 6 \\
\hline 15 & India & EN & 18,39 & 8,16 & 5,22 & 31,77 & N/A \\
\hline 16 & Netherlands & NL & 15,97 & 8,41 & 7,16 & 31,54 & 10 \\
\hline 17 & Latvia & $\mathrm{LV}, \mathrm{EN}$ & 16,43 & 8,28 & 6,76 & 31,47 & 11 \\
\hline 18 & USA & EN & 17,54 & 8,97 & 4,63 & 31,14 & N/A \\
\hline 19 & Singapore & EN & 19,08 & 6,51 & 4,90 & 30,49 & N/A \\
\hline 20 & Estonia & $\mathrm{EE}, \mathrm{EN}$ & 16,81 & 8,66 & 4,94 & 30,41 & 18 \\
\hline 21 & Slovakia & SK, EN & 16,08 & 9,48 & 4,58 & 30,14 & 29 \\
\hline 22 & Luxembourg & EN, FR & 17,17 & 9,19 & 3,61 & 29,97 & 9 \\
\hline 23 & Romania & $\mathrm{RO}, \mathrm{EN}+23$ more* & 15,29 & 10,14 & 3,03 & 28,46 & 8 \\
\hline 24 & Australia & EN & 16,41 & 6,25 & 5,44 & 28,1 & N/A \\
\hline 25 & Germany & $\mathrm{DE}$ & 15,52 & 8,67 & 3,18 & 27,37 & 14 \\
\hline 26 & Norway & NO & 16,48 & 7,52 & 3,33 & 27,33 & 20 \\
\hline 27 & Croatia & CR & 15,09 & 6,51 & 5,72 & 27,32 & 16 \\
\hline 28 & Sweden & SW & 15,61 & 4,94 & 6,42 & 26,97 & 26 \\
\hline 29 & Greece & GR & 16,79 & 6,9 & 3,15 & 26,84 & 15 \\
\hline 30 & Poland & PL, EN & 14,88 & 6,61 & 5,01 & 26,5 & 12 \\
\hline 31 & Liechtenstein & EN, FR, PT, ES & 15,93 & 5,9 & 4,63 & 26,46 & 32 \\
\hline 32 & Iceland & IS, EN & 17,22 & 5,7 & 3,23 & 26,15 & 31 \\
\hline 33 & Japan & JP, EN & 15,1 & 5,96 & 4,96 & 26,02 & N/A \\
\hline 34 & Switzerland & EN & 16,63 & 5,31 & 4 & 25,94 & 24 \\
\hline 35 & Italy & IT & 16,09 & 6,54 & 3,09 & 25,72 & 13 \\
\hline 36 & UK & EN & 15,4 & 6,7 & 3,5 & 25,6 & 22 \\
\hline 37 & Belgium & EN, NL, FR, DE & 15,1 & 6,88 & 3,47 & 25,45 & 17 \\
\hline 38 & Bulgaria & $\mathrm{BG}, \mathrm{EN}$ & 15,88 & 4,89 & 4,29 & 25,06 & 21 \\
\hline 39 & Hungary & $\mathrm{HU}+37$ more* & 15,81 & 5,7 & 3 & 24,51 & 30 \\
\hline 40 & Denmark & DA & 15,58 & 5,48 & 3 & 24,06 & 19 \\
\hline 41 & Czech Republic & $\mathrm{CZ}, \mathrm{EN}$ & 13,45 & 6,15 & 4,19 & 23,79 & 28 \\
\hline 42 & Malta & EN, MT & 12,41 & 6,18 & 4,03 & 22,62 & 23 \\
\hline
\end{tabular}


The lowest results with 3 to 3.15 points were gained by Denmark, Hungary, Romania, Italy and Greece. It is also important to note that such a low overall assessment of this category is due to the fact that 22 out of 42 open data portals do not have the possibility to request a data set. As a result, it impacts both, the "list of open datasets requests" and "involvement in the process" aspects. Thereby the average score of this category is the worst among all analysed - only 5.16 out of 9 points.

As for more detailed results, the best aspects gained more than 2.5 out of 3 points are "thematic categories and tags" (2.64 points), "open data licence" (2.63), "description of dataset" (2.58) and "publisher of dataset" (2.46). However, even these results are not high enough, since this means that the assessment of these aspects is close to "partly fulfilled".

As regards the licence, which was assessed as one of the best aspects, this result is also relevant with (Máchová et al., 2018), where it was found that all portals assessed met this requirement because open data license is today the key part of each portal and all portals are available in machine-readable formats. However, according to (Janssen et al., 2012), only a few years ago, it was a great challenge. Moreover, even now in 2020, there are portals that either do not provide license information or use multiple licenses, where some of them assign limitations on data sets to be used. This aspect is challenging for Hungary, Czech Republic, Germany, Greece, Luxembourg.

The worst aspects for open data portals are the "involvement in the process" (1.54), "list of open datasets requests" (1.55), "user rating and comments" (1.65), "visualization and statistics" (1.77), "forum and contact form" (1.94). An important note here is that 4 out of 5 aspects are related (at least partly) to cooperation between data consumers and users that is one of the most important aspects in the case of open data portal. Moreover, in terms of cooperation and feedback - a total of 7 aspects - only 4 countries scored at least 17.5 out of 21 points - Spain (17.59), Canada (17.9), Cyprus (17.97), Russia (19.88), while a one more country scored more than 17 points - Canada (17.19). However, 6 countries scored less than 10 points (Italy, Switzerland, Bulgaria, Iceland, Hungary and Denmark). This means that improvements must be made as regards to these aspects since they are directly related to the main portals' objective.

A few more examples that demonstrate a positive example of involving data users are: (a) Cyprus and the assessment of user satisfaction through the "satisfactory survey" covering different aspects such as website design, organization/ searchability of data, formats of data sets, API, overall state of open data portal, which, in addition to other opportunities covered in the framework, supports the possibility for users to express their opinion by answering specific questions; (b) Spain, which provides an information on a list of events related to open data which facilitates public engagement, and organize so-called "challenges" to attract users; (c) Canada, which suggests "student challenges". These opportunities supply the abovementioned functionality; therefore, these additional aspects highlight these portals among others.

As for "thematic categories and tags", the majority of open data portals allocates data sets to different categories that mostly follows the same guidelines in regards of the nature of these categories, and the most portals provides a tags that are separated from categories. However, even this aspect sometimes is challenging. For instance, for the Latvia's open data portal, despite 14 different categories exist, by the end of May 2020 there were 102 data sets not belonging to any category, that is not related to poor design, because the publisher did not specify a category that should be assigned to the specific data set (Nikiforova, 2020a). As a result, the absence of the allocation to the specific category makes it difficult to find data sets if a user studies data sets by category. Some countries solve this challenge by involving the category "other" or "uncategorized", as in the case of Bulgaria.

As regards visualisation and statistics by which in (Máchová et al., 2018) are meant visualization and analytical possibilities for obtaining information about a data set, such as charts or visualizations in maps, this aspect is really weak, since it is assessed by 1.77 points (worst aspect for the $1^{\text {st }}$ category) - sometimes the possibility of visualizing data is provided, but it is often ensured as a preview feature for data sets. The best results belong to Cyprus and Singapore scoring more than 2.8 points.

As for the "user rating and comments", this is often not provided, however, there are countries that provide a "good example" to others that should be considered and implemented. These countries are France, Cyprus, Austria, Romania, Canada and Taiwan, since the participants assessed this aspect very high.

As regards multilingualism ( $4^{\text {th }}$ column in Table 2), that, according to (Zuiderwijk et al., 2015), is one of success factors of the open data initiatives since this facilitates the reuse of data (Attard et al., 2015), 33.33\% of the portals analysed are available in only one language and only $42.86 \%$ of them are available in English, while other portals are available in only one country language, thereby reducing the number of potential users. In addition, it should also be noted that even if the portal is available in more than one language, only 
part of its content is sometimes translated, but most of the information is still provided in the main language. For some countries this may not have a serious impact, however, such "big players" as Germany, Denmark, Austria etc., may lose potential users. The worst results in the way of languages supported belong to Germany and Denmark, where portals support only relevant languages and are not suitable for English speakers, thereby limiting the portal's capabilities and the reuse of their data sets. Slovenia could be mentioned here as one of the unusual positive examples, since despite the fact that the portal is provided in only Slovenian, the portal holders provide a built-in Google Translate that allows the content of the entire portal to be adapted. In some cases, this option is even better than translation in several languages, since, as demonstrate the Romanian and Hungarian portals, in the case of attempts to provide as many languages as possible (35+), the most part of the content is not translated (e.g., name of data sets, their description, etc.).

One more aspect, that is considered as one of the key success factors for open data portals, is the presence of use-cases or showcases, by which are meant open data based applications published or at least mentioned in portal, thereby enabling users to get an insight of the potential of open data (Zuiderwijk et al., 2015). For the study carried out, 30 out of the 42 open data portals analysed (71.43\%) provides showcases, which number may range from 3 (Slovakia) to 2044 (France). In addition, in some cases, such as France, Luxembourg or Portugal, the national portal has a "use case upload" feature. Moreover, 18 portals offer a mapping between the use cases and the data sets that these use cases are based on (for instance, Ireland), that should impact an insight of the potential of open data even better, thereby increasing public participation.

To sum up, Cyprus, France, Russia, Spain, Taiwan, Canada, Austria, Colombia, New Zealand, Ireland, Portugal demonstrated the best results, according to the 40 participants, while the portals of Malta, the Czech Republic, Denmark, Hungary, Bulgaria and Belgium are currently less successful in terms of their usability. This is partly in line with (Blank, 2019), however, as shown in Table 2, the results vary significantly for some countries, and this study shows some aspects that are most affected by such assessment. Thereby, their improvement would have an impact on the overall level of satisfaction of these portals, however, possible improvements can be obtained from the positive examples listed.

\section{CONCLUSION}

The popularity of open [government] data in recent decades has been growing more rapidly. Governments are moving toward e-governments, and OGD aims to facilitate these changes. However, it is well-known fact that open data by itself is not of great importance since value is generated by the use of data. It is therefore important to establish cooperation between data publishers and data consumers, that usually is done through a platform that can provide faster, better and different data usage opportunities. In other words, in order to benefit from the data being released and opened, a sufficiently high-quality and user-friendly open data portal should be provided, allowing access to the data for all stakeholders, which would result in the transformation of the data into value and knowledge for the society. This study addresses one of the most important aspects - the usability of open data portals.

42 national open data portals were analysed by applying to them a single common framework allowing their comparative analysis to be carried out. The analysis carried out demonstrates that there are portals that can be considered as leaders and as an example for the less successful open data portals that were also identified. However, this study demonstrates that even more successful open data portals have the room for improvements. In addition, the study shows that, in cases of less successful portals, certain aspects are sometimes better implemented.

This study highlights the weakest aspects for 42 national open data portals analysed, pointing on both, the most common weakest points, and individual. This should allow national open data portals to improve the usability of their portals, in particular, since 4 out of 5 weakest aspects are related (at least partly) to feedback and cooperation between data consumers and users.

It is intended to extend the results provided to a more detailed coverage of portals and individual aspects in order to provide comprehensive overview of the state of play of the open data portals. In addition, it is recommended that countries analyse their portals in depth, thereby extending this study, since some aspects should be covered by citizens of the country, as demonstrated in (Nikiforova, 2020a), by providing a set of aspects covered by the framework used with additional aspects, as well as by analysing data sets. 


\section{ACKNOWLEDGMENT}

This work has been supported by University of Latvia Faculty of Computing project AAP2016/B032 "Innovative information technologies". I am also grateful to the participants for taking part in the experiment within my workshop "Open data and data quality", which allowed to collect data and led to such results.

\section{REFERENCES}

Attard, J. et al. (2015). A systematic review of open government data initiatives. Government Information Quarterly, $32(4), 399-418$.

Blank, M. (2019) Open Data Maturity Report 2019, Europeam Data Portal, online: https://www.europeandataportal.eu/sites/default/files/open_data_maturity_report_2019.pdf

Charalabidis, Y. et al. (2018). The Open Data Landscape. In The World of Open Data (pp. 1-9). Springer, Cham.

Faini, F. and Palmirani, M. (2016). Italian Open and Big Data Strategy. In International Conference on Electronic Government and the Information Systems Perspective (pp. 105-120). Springer, Cham.

Hansen, H. S. and Schrøder, L. (2019). The Societal Benefits of Open Government Data with Particular Emphasis on Geospatial Information. In International Conference on Electronic Government and the Information Systems Perspective (pp. 31-44). Springer, Cham.

Janssen, M. et al. (2012). Benefits, adoption barriers and myths of open data and open government. Information systems management, 29(4), 258-268.

Máchová, R. et al. (2018). Usability evaluation of open data portals. Aslib Journal of Information Management.

Matheus, R. et al. (2012). Anti-corruption online monitoring systems in Brazil. In Proceedings of the 6th International Conference on Theory and Practice of Electronic Governance (pp. 419-425).

McBride, K. et al. (2018). Open Government Data Driven Co-creation: Moving Towards Citizen-Government Collaboration. In International Conference on Electronic Government (pp. 184-195). Springer.

Nikiforova, A. (2020a). Assessment of Latvia's Open Data Portal or how Close are We to Gaining Benefits from Open Data (in preparation)

Nikiforova, A. (2020b). Definition and Evaluation of Data Quality: User-oriented Data Object-Driven Approach to Data Quality Assessment. Baltic Journal of Modern Computing, 8(3).

Nikiforova, A. (2019). Analysis of Open Health Data Quality Using Data Object-Driven Approach to Data Quality Evaluation: Insights from a Latvian Context, In IADIS International Conference e-Health 2019, pp.119-126, MCCSIS 2019. IADIS.

Osagie, E. et al. (2017). Usability evaluation of an open data platform. In Proceedings of the 18th Annual International Conference on Digital Government Research (pp. 495-504).

Purwanto, A. et al. (2020). Citizen engagement with open government data. Transforming Government: People, Process and Policy.

Susha, I. et al. (2015). Benchmarks for evaluating the progress of open data adoption: Usage, limitations, and lessons learned. Social Science Computer Review, 33(5), 613-630.

Tinholt, D. (2013). The Open Data Economy: Unlocking Economic Value by Opening Government and Public Data. Capgemini Consulting.

Umbrich, J. et al. (2015). Quality assessment and evolution of open data portals. In 20153 rd international conference on future internet of things and cloud (pp. 404-411). IEEE.

UNDESA, U. (2016). Department of Economic and Social Affairs. United Nations e-government survey 2016: E-government in support of sustainable development.

Zuiderwijk, A. et al. (2015). Open data disclosure and use: Critical factors from a case study. In CeDEM15: Conference for E-Democracy and Open Government (Vol. 197). MV-Verlag. 